\title{
Uso de Resina de Intercambio Aniónico para la Recuperación del Complejo Oro Tiosulfato desde Soluciones Acuosas
}

\author{
Miriam E. Chaparro(1), Jesús L. Valenzuela ${ }^{(2)}$, Guillermo T. Munive ${ }^{(2)}$ y José R. Parga ${ }^{(3)}$ \\ (1) Instituto de Ingeniería, Universidad Autónoma de Baja California, Blvd. Benito Juárez y Calle a \\ la Normal S/N, Col. Insurgente Este, Mexicali, B.C.-México. (miriam_edith1981@hotmail.com). \\ (2) Departamento de Ingeniería Química y Metalurgia, Universidad de Sonora, \\ Rosales y Blvd. Luis Encinas, Col. Centro, Hermosillo, Sonora-México. (jvalen@iq.uson.mx). \\ (3) Departamento de Metalurgia y Materiales, Instituto Tecnológico de Saltillo, Blvd. Venustiano \\ Carranza \# 2400, Saltillo, Coahuila-México. (jrparga@its.mx).
}

Recibido Ago. 26, 2011; Aceptado Nov. 02, 2011; Versión Final recibida Dic. 26, 2011

\begin{abstract}
Resumen
Se estudió la adsorción de oro utilizando la resina AuRIX ${ }^{\circledR} 100$ en medio tiosulfato de amonio, evaluando algunas variables que afectan la cinética del proceso tales como: temperatura, velocidad de agitación, $\mathrm{pH}$, concentración de tiosulfato de amonio $\left(\mathrm{NH}_{4}\right)_{2} \mathrm{~S}_{2} \mathrm{O}_{3}$ y concentración de oro. El estudio se llevó a cabo en un reactor batch y una columna de intercambio iónico. Las condiciones de operación que presentaron mejores resultados de extracción de oro a $25^{\circ} \mathrm{C}$, fueron: $\mathrm{pH}=10.5$, velocidad de agitación $=500 \mathrm{rpm},[\mathrm{Au}]=1 \mathrm{mg} / \mathrm{l},\left[\left(\mathrm{NH}_{4}\right)_{2} \mathrm{~S}_{2} \mathrm{O}_{3}\right]=0.04 \mathrm{M}, 5$ gramos de resina. Los resultados indican que al aumentar $\left[\left(\mathrm{NH}_{4}\right)_{2} \mathrm{~S}_{2} \mathrm{O}_{3}\right.$ ] favorece la adsorción en un 99\% durante tres horas, siendo afectado notablemente por la presencia de amonio. Las condiciones con mejores resultados en la columna a $25^{\circ} \mathrm{C}$ fueron, $\mathrm{pH}=10.5 \mathrm{y}[\mathrm{Au}]=1 \mathrm{mg} / \mathrm{l}$.
\end{abstract}

Palabras clave: adsorción oro, tiosulfato, resina AuRIX ${ }^{\circledR} 100$, cinética

\section{Use of Anion Exchange Resin for the Recovery of the Complex Gold Thiosulfate from Aqueous Solutions}

\begin{abstract}
The adsorption and elution of gold in thiosulfate-ammonia media were studied using the resin AuRIX ${ }^{\circledR} 100$, evaluating some variables that affect the kinetics of the process, such as: temperature, stirring speed, $\mathrm{pH}$, thiosulfate concentration $\left(\mathrm{NH}_{4}\right)_{2} \mathrm{~S}_{2} \mathrm{O}_{3}$ and gold concentration. The study was carried out in a batch reactor and an ion exchange column. The operation conditions that presented better results of gold extraction at $25^{\circ} \mathrm{C}$ were: $\mathrm{pH}=10.5$, stirring speed $=500 \mathrm{rpm}$, $[\mathrm{Au}]=1 \mathrm{mg} / \mathrm{l},\left[\left(\mathrm{NH}_{4}\right)_{2} \mathrm{~S}_{2} \mathrm{O}_{3}\right]=0.04 \mathrm{M}$, and $5 \mathrm{grams}$ of resin. The results indicate that by increasing $\left[\left(\mathrm{NH}_{4}\right)_{2} \mathrm{~S}_{2} \mathrm{O}_{3}\right]$ favors the adsorption by $99 \%$ during three hours, being noticeably affected by the presence of ammonia. The conditions with results on the column at $25^{\circ} \mathrm{C}$ were, $\mathrm{pH}=10.5,[\mathrm{Au}]=1$ $\mathrm{mg} / \mathrm{l}$.
\end{abstract}

Keywords: gold adsorption, thiosulfate, $A u R I X^{\circledR} 100$ resin, kinetics 


\section{INTRODUCCIÓN}

La mayoría de las plantas minero metalúrgicas utilizan el proceso de cianuración para la extracción de oro, pero el principal problema es su alta toxicidad, por lo que en recientes años, se han desarrollado métodos alternativos para ello, como es el caso del tiosulfato de amonio y tiourea, que son tan efectivos como el cianuro, pero que aun no han logrado aplicaciones industriales ya que presenta dificultades inherentes a la química de la solución y los métodos para su recuperación han limitado su progreso (Aylmore et al, 2001). Además, las disoluciones de lixiviación han sido realizadas por Adsorción en carbón activado, extracción por solventes, precipitación por el proceso Merrill-Crowe, electrodeposición y resinas de intercambio iónico. Estudios recientes (Chaparro, 2008), demostraron la viabilidad de la extracción del complejo oro tiosulfato, tratándolo con guanidina sobre una resina de intercambio aniónica. Por otro lado, la lixiviación de oro desde concentrados y/o minerales usando disoluciones de tiosulfato y amoniaco han sido estudiadas como alternativas al proceso de cianuración. Sin embargo, uno de los problemas asociados al uso del tiosulfato es la recuperación de oro desde las disoluciones obtenidas ya que son catalizadas por cobre y amoniaco, presentando un posible mecanismo de reacción, como el observado en las ecuaciones 1 y 2.

$$
\begin{aligned}
& \mathrm{Au}+\mathrm{Cu}\left(\mathrm{NH}_{3}\right)_{4}^{2}+4 \mathrm{~S}_{2} \mathrm{O}_{3}^{2-} \rightarrow \mathrm{Au}\left(\mathrm{S}_{2} \mathrm{O}_{3}\right)_{2}^{3-}+\mathrm{Cu}\left(\mathrm{S}_{2} \mathrm{O}_{3}\right)_{2}^{3-}+4 \mathrm{NH}_{3} \\
& \mathrm{Cu}\left(\mathrm{S}_{2} \mathrm{O}_{3}\right)_{2}^{3-}+\mathrm{O}_{2}+2 \mathrm{H}_{2} \mathrm{O}+6 \mathrm{NH}_{3} \rightarrow 4 \mathrm{Cu}\left(\mathrm{NH}_{3}\right)_{4}^{2+}+8 \mathrm{~S}_{2} \mathrm{O}_{3}^{2-}+4 \mathrm{OH}^{-}
\end{aligned}
$$

Con respecto al uso de resinas para la recuperación de oro desde disoluciones acuosas con tiosulfato y amoniaco, existen estudios en literatura (Molleman et al, 2002), donde utilizan resinas de base débil y la especie estable que se adsorbe es el complejo oro-tiosulfato, existiendo aniones en la disolución acuosa, como sulfito, tritionato y tetrationato que son adsorbidos por la resina, provocando una disminución en el complejo $\mathrm{Au}\left(\mathrm{S}_{2} \mathrm{O}_{3}\right)_{2}^{3-}$. Del mismo modo, en investigaciones realizadas (Aguayo, 2007) sobre la extracción por solventes para Ag y Au en soluciones alcalinas provenientes de cianuración, se utilizó como extractante LIX 79 en base guanidina e igualmente que AuRIX ${ }^{\circledR} 100$ en soluciones de tiosulfato y como diluyente keroseno, determinando que el complejo oro-cianuro, se extrae a $10.5<\mathrm{pH}<11.2$. Al agregar tridecanol $5 \%$ en volumen, obteniendo como resultado una adecuada selectividad con $10 \%$ LIX 79. Lo anterior, fue generado a partir de licores de lixiviación en columna con minerales oxidados y sulfurados pero conteniendo $\mathrm{Ag}$ y pequeñas cantidades de $\mathrm{Au}, \mathrm{Cu}$ y $\mathrm{Zn}$. Sin embargo, es necesario realizar una exhaustiva investigación utilizando el complejo oro-tiosulfato. Así mismo, en investigaciones de adsorción de Au (Navarro et al, 2006), utilizando resina Amberlita IRA-410 en medio tiosulfato-amoniaco, se demostró que la adsorción de Au es rápida, debido a la presencia de tiosulfato esté desfavoreció la adsorción; sin embargo, este aspecto no queda muy claro. De tal manera, que estudios realizados para evaluar la eficiencia de la resina AuRIX ${ }^{\circledR} 100$ (Valenzuela et al, 2006), para la recuperación del complejo oro-cianuro a $\mathrm{pH}$ 10.7, se determino que al aumentar la relación sólidolíquido la cantidad de Au por unidad de masa de resina aumenta proporcionalmente. Durante la adsorción de $\mathrm{Au}$ en carbón activado, se ha discutido que el complejo oro-tiosulfato no es adsorbido eficientemente, pero no obstante a ello (Vargas et al, 2006), ha reportado que la presencia de tiosulfato, amoniaco e impurezas $(\mathrm{Cu}, \mathrm{Zn})$, son perjudiciales para la adsorción de Au ya que la $\mathrm{Ea}=9.13 \mathrm{~kJ} / \mathrm{mol}$; por lo contrario, el complejo oro-cianuro si es adsorbido por el carbón activado. Por otro lado, se han evaluado materiales (Seob et al, 2010) para la adsorción de Au obteniendo $427.77 \mathrm{mg} / \mathrm{g}$ con resina Amberjet $^{\mathrm{TM}} 4400,170.64 \mathrm{mg} / \mathrm{g}$ en carbón activado y 361.76 $\mathrm{mg} / \mathrm{g}$ en medio biosorbente. Por otra parte (Munive et al, 2011), se realizaron estudios comparativos de lixiviación sobre un mineral refractario conteniendo cantidades de sulfuros, pirita y pirrotita, en presencia de cianuro y tiosulfato, donde este último permanece estable, pero con la finalidad de recuperar $\mathrm{Au}$ y $\mathrm{Ag}$ durante $48 \mathrm{hrs}$ y ajustando el pH menor a 9.6 con solución de $\mathrm{NaOH}$ y $\mathrm{Ca}(\mathrm{OH})_{2}$. Resultados obtenidos (Breuer et al, 2002), donde el tiosulfato disuelve al cobre presente en el mineral, se muestra que favorece al proceso, ya que actúa como oxidante, provocando que la disolución de $\mathrm{Au}$ y Ag disminuya, atribuido a la inestabilidad del complejo cuprotetramina (Yen et al, 1998) a pH=9.5. De lo anterior, se puede observar que existe un gran interés por proponer lixiviantes alternativos al cianuro para la adsorción de Au, con compuestos 
menos tóxicos y buscando técnicas viables que permitan obtener una máxima adsorción de metales precisos. En la Tabla 1. Se enlistan las condiciones de lixiviación de diferentes minerales. Observando que la resina AuRIX ${ }^{\circledR} 100$ contiene el componente activo guanidina, utilizada para soluciones de lixiviación del complejo oro-tiosulfato de amonio, constituida por una red de macro retículas de estireno y divinil-benceno que funcionalizada con grupos guanidina actúan como bases orgánicas fuertes.

Tabla 1. Comparación de parámetros de lixiviación en minerales y avances en procesos de adsorción de oro

\begin{tabular}{|c|c|c|c|c|c|c|c|}
\hline Antecedentes & Tipo de Mineral & $\begin{array}{c}\mathrm{T} \\
\left({ }^{\circ} \mathrm{C}\right)\end{array}$ & $\mathrm{pH}$ & $\begin{array}{l}\text { Ret } \\
\text { (h) }\end{array}$ & $\begin{array}{l}\mathrm{S}_{2} \mathrm{O}_{3}^{2-} \\
(\mathrm{M})\end{array}$ & $\begin{array}{l}\mathrm{NH}_{3} \\
(\mathrm{M})\end{array}$ & $\begin{array}{l}\% \text { de } \\
\text { Rec. }\end{array}$ \\
\hline Yen et al, (1998) & $\begin{array}{c}\text { Au-Cu-0.36\% Cu, } \\
\text { Au: } 7.2-7.9 \text { g/ton }\end{array}$ & $\bar{A}$ & 10 & 24 & 0.5 & 6 & $95-97$ \\
\hline Molleman et al, (2002) & Concentrado de Pirita & 35 & $10-11$ & 6 & 0.05 & 0.4 & 83 \\
\hline Navarro et al, (2002) & Conc. de Au: 95 g/ton & 25 & $9-10$ & 10 & 0.3 & - & 94 \\
\hline Breuer et al, (2002) & $\mathrm{Cu}(\mathrm{II}), \mathrm{Au}$ & $30-50$ & $8-9.4$ & - & 0.2 & $0-0.6$ & - \\
\hline Ficeriová et al, (2002) & Conc. de CuPbZn & 25 & $6-7$ & 2 & 0.5 & - & 99 \\
\hline Zhang et al, (2002) & 20 mg/l, Au: 2 g/ton & $23-25$ & 11 & 24 & 0.1 & 0.2 & $80-90$ \\
\hline Valenzuela et al, (2006) & $\begin{array}{c}\text { Au: } 0.32, \\
\text { Ag: } 0.43, \mathrm{Cu}: 6.2\end{array}$ & 25 & $8-10.7$ & 3 & - & - & 98 \\
\hline Navarro et al, (2006) & Au: $8 \mathrm{mg} / \mathrm{l}$ & 25 & $9-11$ & 3 & $0-0.5$ & $0-0.5$ & $92-98$ \\
\hline Vargas et al, (2006) & Au: 10 mg/l, 2.2 g/ton & 25 & 9.5 & 4 & $0-0.50$ & $1-4$ & $15-28$ \\
\hline Jeffrey et al, (2010) & $10 \mathrm{mg} / \mathrm{l}$ & $25-60$ & 9 & 24 & - & $5-100 \mathrm{nM}$ & 2.7 \\
\hline Seob et al, (2010) & $\mathrm{Au}$ & $25 \pm 2$ & $1-6$ & $2-3$ & - & 0.1 & $84-96$ \\
\hline Munive et al, (2011) & Au: $3.15 \mathrm{~g} /$ ton & 25 & 9.5 & 48 & 0.25 & 0.4 & $70-82$ \\
\hline
\end{tabular}

Por lo anteriormente expuesto, en este trabajo se propone estudiar el proceso de adsorción de Au, mediante pruebas batch y en columna, evaluando la eficiencia de la resina de intercambio aniónico AuRIX ${ }^{\circledR} 100$ para la adsorción del complejo oro-tiosulfato en medio amoniaco-tiosulfato, con el objetivo de obtener los parámetros cinéticos que intervienen en la reacción, evaluando el contenido de Au a un tiempo determinado, dando seguimiento a la reacción en las soluciones por absorción atómica con la finalidad de establecer un proceso como método alternativo para el uso de las resinas de intercambio iónico.

\section{METODOLOGÍA}

El método seguido para el desarrollo del presente estudio, consistente en la extracción de oro mediante el uso de guanidina, se utiliza como componente activo guanidina en la resina AuRIX ${ }^{\circledR} 100$, determinando además la influencia de las variables que intervienen en la reacción durante la extracción del metal.

Extracción de oro utilizando una resina AuRIX ${ }^{\circledR} 100$, pruebas batch

En un reactor batch Kettles marca Pyrex, capacidad $500 \mathrm{ml}$, con disolución acuosa de $250 \mathrm{ml}$, con parámetros experimentales: Tiempo de contacto $=3 \mathrm{~h}$, temperatura $=25^{\circ} \mathrm{C},[\mathrm{Au}]_{\mathrm{i}}=1-8 \mathrm{mg} / \mathrm{l}$, razón resina/disolución= $20000 \mathrm{mg} / \mathrm{l}$, velocidad de agitación= 200-800 rpm. Evaluando el porcentaje de oro adsorbido en función del tiempo. Las variables se presentan en la Tabla 2. Agregando al reactor la disolución acuosa, $[\mathrm{Au}]_{i},\left[\left(\mathrm{NH}_{4}\right)_{2} \mathrm{~S}_{2} \mathrm{O}_{3}\right]$, ajustando la temperatura y pH, se agregó la resina a la disolución y se agitó el sistema, se sacaron muestras de la fase acuosa de $10 \mathrm{ml}$ cada 30 min para análisis, se midió el pH, en 3 h se detuvo la agitación, se separaba la resina de la disolución acuosa y para su análisis por la técnica de absorción atómica en un espectrofotómetro Perkin Elmer 3110 y la carga de oro en la resina se determinó por balance de oro.

Extracción de oro utilizando una resina AuRIX ${ }^{\circledR} 100$, pruebas en columna

Las pruebas en columna de intercambio iónico dividida en cuatro secciones conectadas en serie separación sólido-líquido, con una suspensión acuosa y resina en cada etapa, cada sección tiene 
una malla de alambre en la parte inferior y superior para retener la resina colocada en cada una de las celdas. Las dimensiones de las celdas son de $2.5 \times 7.7 \mathrm{~cm}$ de diámetro y altura $(2.54 \times$ $7.52 \mathrm{~cm}$ ), para pasar la solución conteniendo oro, a través de la columna se usó una bomba peristáltica en tres corridas experimentales, con 5 gramos de resina como fase fija a $25^{\circ} \mathrm{C}$, con una velocidad de $0.50,0.60,0.70 \mathrm{rpm}$, con $7.43 \mathrm{ml} / \mathrm{min}, 15 \mathrm{ml} / \mathrm{min}, 23 \mathrm{ml} / \mathrm{min}$, equivalente a 89 $276 \mathrm{BVH}$, fue necesario saber la relación flujo de solución/volumen de resina y también fueron analizadas las isotermas de adsorción.

Disolución de oro y disolución eluyente

La disolución de oro se llevo a cabo utilizando agua destilada y deionizada y tiosulfato de amonio $\left(\mathrm{NH}_{4}\right)_{2} \mathrm{~S}_{2} \mathrm{O}_{3}$ con pureza 99\% marca Aldrich, la solución de oro $(1000 \pm 2 \mu \mathrm{g} / \mathrm{ml})$, se ajusto el pH con $\mathrm{NH}_{4} \mathrm{OH}$ y en la elución con hidróxido de sodio [ $\mathrm{NaOH}$, sulfato de cobre pentahidratado [CuSO $\left.{ }_{4} \cdot 5 \mathrm{H}_{2} \mathrm{O}\right]$, perclorato de sodio $\left[\mathrm{NaClO}_{4} \cdot 2 \mathrm{H}_{2} \mathrm{O}\right]$, sulfito de sodio $\left[\mathrm{Na}_{2} \mathrm{SO}_{3}\right]$ y cloruro de sodio $[\mathrm{NaCl}]$.

\section{Pruebas de desorción}

Los experimentos de desorción se realizaron en un reactor de $500 \mathrm{ml}$ de capacidad con agitación, donde se mantuvieron constantes las siguientes condiciones experimentales: $\mathrm{pH}=$ 9.5-11.5, concentración de $\mathrm{ClO}_{4}^{-}$, concentración de $\mathrm{SO}_{3}^{2-}$, concentración de $\mathrm{Cr}$, concentración de $\mathrm{NaOH}$.

Caracterización de la resina AuRIX ${ }^{\circledR} 100$

La caracterización se llevó a cabo por análisis difracción de rayos-X, caracterización por análisis infrarrojo y microscopio electrónico de barrido (MEB).

Tabla 2: Variables y rangos de experimentación en la etapa de adsorción

\begin{tabular}{lc}
\hline Variable & Rango de experimentación \\
\hline $\mathrm{pH}$ & $9-10.50$ \\
Concentración de $\mathrm{Au}_{\mathrm{i}}$ & $1-8 \mathrm{mg} / \mathrm{l}$ \\
Concentración de $\left(\mathrm{NH}_{4}\right)_{2} \mathrm{~S}_{2} \mathrm{O}_{3}$ & $0.00674-0.04 \mathrm{M}$ \\
\hline
\end{tabular}

\section{RESULTADOS Y DISCUSIÓN}

En las Fig. 1 a 3 se estudió el efecto de tiempo de contacto en la extracción de oro, se ve el efecto del cambio de concentraciones de $1 \mathrm{mg} / \mathrm{l}$ a $8 \mathrm{mg} / \mathrm{l}$, al aumentar el $\mathrm{pH}$ se logró un aumento en porcentaje extracción, donde el pH mejor fue de 10.5, en 3 h. En la Fig. 4 se muestra el efecto de la velocidad de agitación en la extracción de oro en resina AuRIX ${ }^{\circledR} 100$.

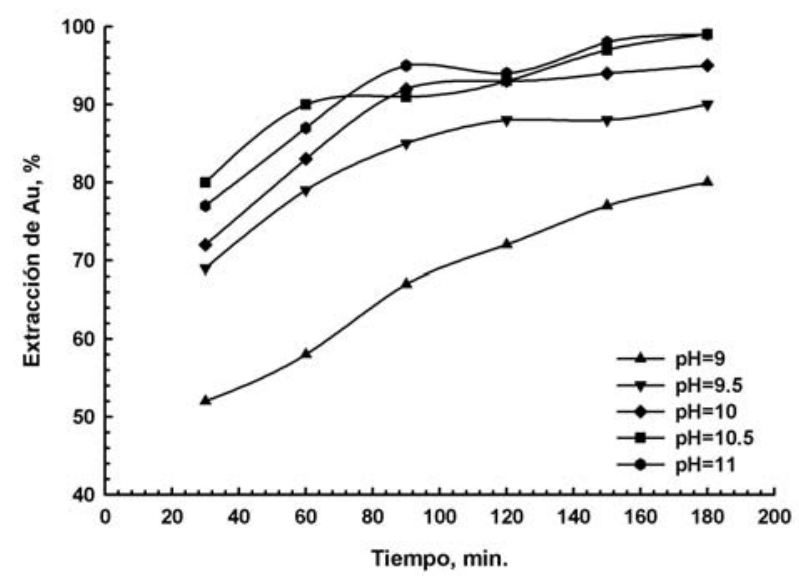

Fig. 1: Extracción de oro en resina AuRIX ${ }^{\circledR} 100$. Condiciones: $\left[\left(\mathrm{NH}_{4}\right)_{2} \mathrm{~S}_{2} \mathrm{O}_{3}\right]=0.04 \mathrm{M},[\mathrm{Au}]_{i}=1 \mathrm{mg} / \mathrm{l}$, AuRIX ${ }^{\circledR} 100=5 \mathrm{~g}$. y $T=25^{\circ} \mathrm{C}$

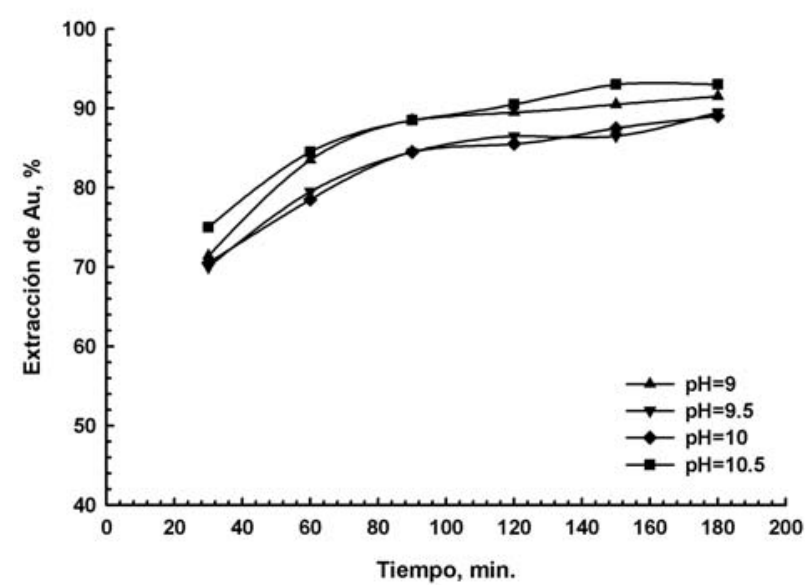

Fig. 2: Extracción de oro en resina AuRIX ${ }^{\circledR} 100$. Condiciones: $\left[\left(\mathrm{NH}_{4}\right)_{2} \mathrm{~S}_{2} \mathrm{O}_{3}\right]=0.04 \mathrm{M},[\mathrm{Au}]_{\mathrm{i}}=2 \mathrm{mg} / \mathrm{l}$, AuRIX $^{\circledR} 100=5 \mathrm{~g}$, velocidad $=500 \mathrm{rpm}$ y $\mathrm{T}=25^{\circ} \mathrm{C}$ 


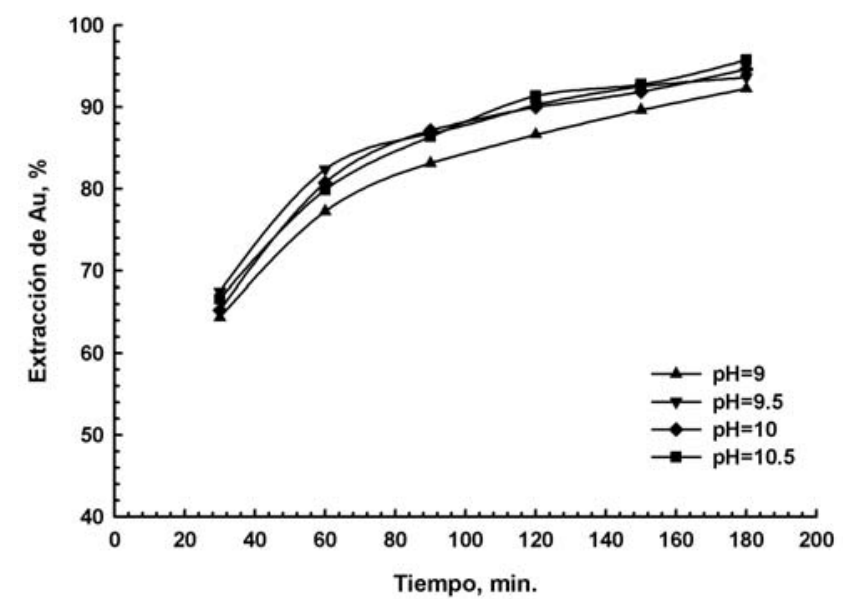

Fig. 3: Efecto de tiempo de contacto en la extracción de oro en resina AuRIX ${ }^{\circledR} 100$.

Condiciones: $\left[\left(\mathrm{NH}_{4}\right)_{2} \mathrm{~S}_{2} \mathrm{O}_{3}\right]=0.04 \mathrm{M},[\mathrm{Au}]_{i}=8 \mathrm{mg} / \mathrm{l}$, AuRIX ${ }^{\circledR} 100=5 \mathrm{~g}, \mathrm{pH}=9-10.5$ y $\mathrm{T}=25^{\circ} \mathrm{C}$

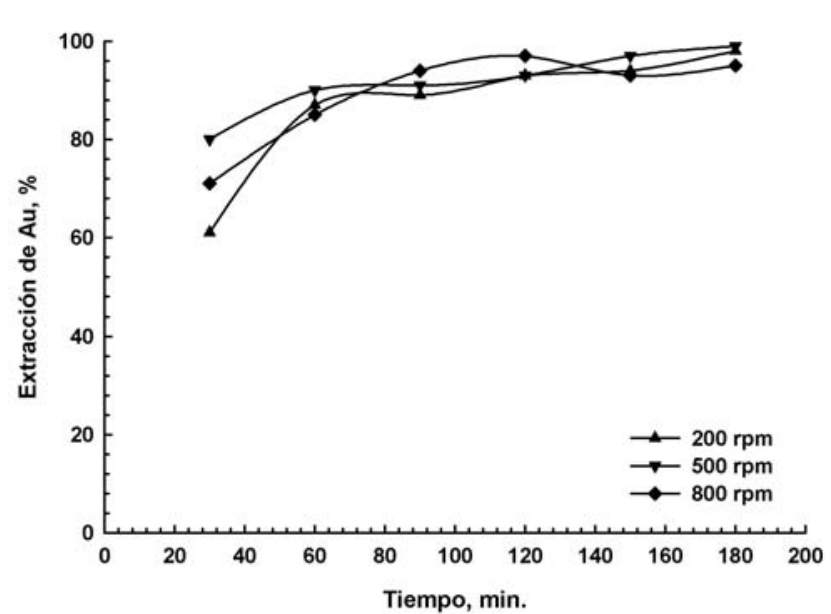

Fig. 4: Efecto de velocidad de agitación en la extracción de oro en resina AuRIX ${ }^{\circledR} 100$. Condiciones: $\left[\left(\mathrm{NH}_{4}\right)_{2} \mathrm{~S}_{2} \mathrm{O}_{3}\right]=0.04 \mathrm{M},[\mathrm{Au}]_{i}=1 \mathrm{mg} / \mathrm{l}, \mathrm{AuRIX}{ }^{\circledR} 100=5 \mathrm{~g}$, $\mathrm{pH}=10.5$, velocidad $=200,500,800 \mathrm{rpm}$

En la Fig. 5 se muestra el efecto de pH en la extracción de oro en la resina. Se encontró que la mayor extracción de oro se realizó a $\mathrm{pH}=10.5$ y 120 minutos y en la Fig. 6 se observó el efecto de la $\left[\left(\mathrm{NH}_{4}\right)_{2} \mathrm{~S}_{2} \mathrm{O}_{3}\right]$ en la adsorción de oro en la resina al trabajar a pH 10.5. Es evidente el efecto positivo que produce el contenido de tiosulfato en la disolución acuosa sobre el porcentaje de oro adsorbido, en $3 \mathrm{~h}$. En presencia de tiosulfato, la adsorción de oro alcanzó un 99\% y con 0.00674 $\mathrm{M}$ de tiosulfato el comportamiento no fue estable, la adsorción disminuyó a 98\%, $0.03 \mathrm{M}$ de tiosulfato la adsorción disminuyó a 98\% y 0.04 M de tiosulfato la adsorción aumento a 99\%.

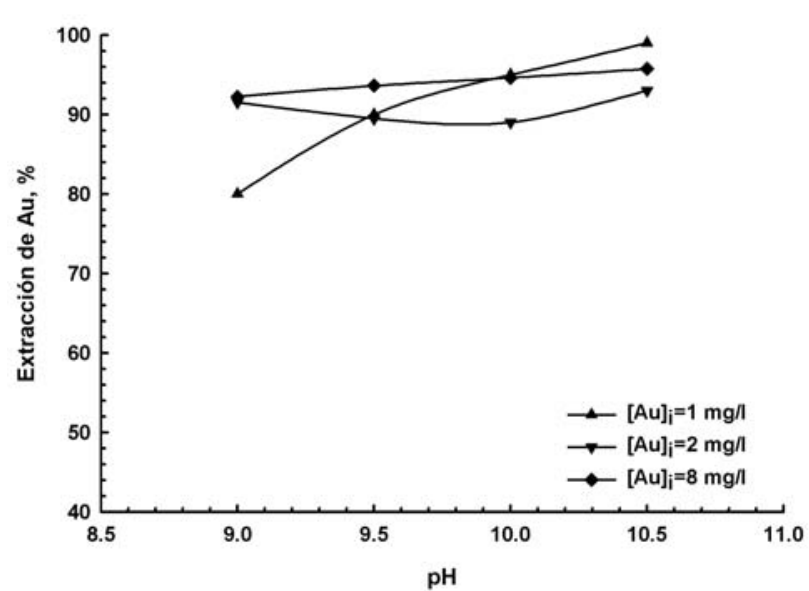

Fig. 5: Efecto de pH en extracción de oro en resina AuRIX ${ }^{\circledR} 100$. Condiciones: $\left[\left(\mathrm{NH}_{4}\right)_{2} \mathrm{~S}_{2} \mathrm{O}_{3}\right]=0.04 \mathrm{M}$, $[\mathrm{Au}]_{\mathrm{i}}=1,2,8 \mathrm{mg} / \mathrm{l}, \mathrm{AuRIX}{ }^{\circledR} 100=5 \mathrm{~g}, \mathrm{pH}=9-10.5 \mathrm{y}$ $\mathrm{T}=25^{\circ} \mathrm{C}$

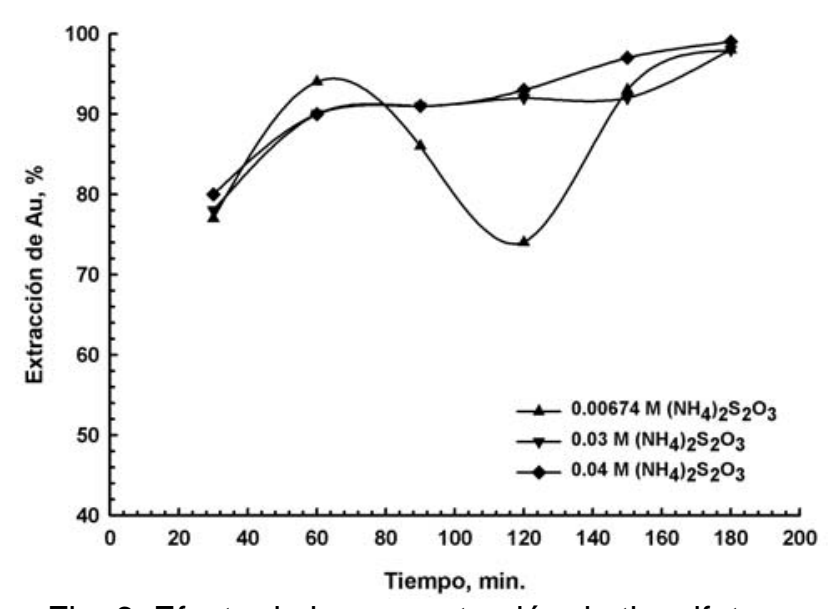

Fig. 6: Efecto de la concentración de tiosulfato en extracción de oro en resina AuRIX ${ }^{\circledR} 100$.

Condiciones: $\left[\left(\mathrm{NH}_{4}\right)_{2} \mathrm{~S}_{2} \mathrm{O}_{3}\right]=0.00674,0.03,0.04 \mathrm{M} \mathrm{y}$ $[\mathrm{Au}]_{\mathrm{i}}=1 \mathrm{mg} / \mathrm{l}$, AuRIX ${ }^{\circledR} 100=5 \mathrm{~g}$, velocidad $=500 \mathrm{rpm}$ y $\mathrm{T}=25^{\circ} \mathrm{C}$

Se realizaron tres corridas experimentales con 5 gramos de resina, con una velocidad de 0.50, $0.60,0.70 \mathrm{rpm}$, con $7.43 \mathrm{ml} / \mathrm{min}, 15 \mathrm{ml} / \mathrm{min}, 23 \mathrm{ml} / \mathrm{min}$, equivalente a 89, 180 y $276 \mathrm{BVH}$. Los resultados se muestran en las Fig. 7 a 10.

En la Fig. 7 Muestra el perfil de la velocidad de adsorción, en cada sección de la columna, observándose que el trabajo en la última es inapreciable, pero en la primer sección es más rápida, por lo que esta será la primera que llegue a saturación, se observó que con el paso del tiempo la concentración que sale de cada sección va en aumento, en el momento que la concentración que entre sea igual a la que sale. 


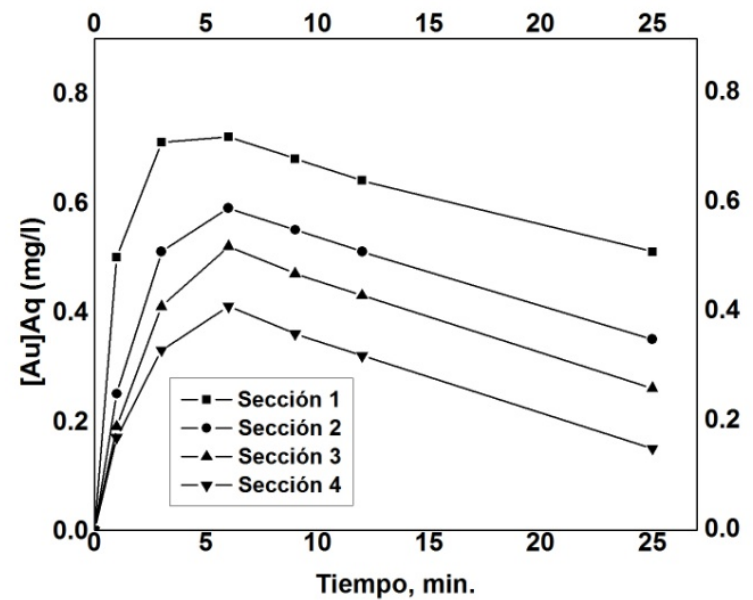

Fig. 7: Perfil en la extracción de oro al utilizar resina AuRIX ${ }^{\circledR} 100$, en las secciones de la columna con velocidad de 0.50 , con flujo de $7.43 \mathrm{ml} / \mathrm{min}$, equivalente a $89 \mathrm{BVH}$

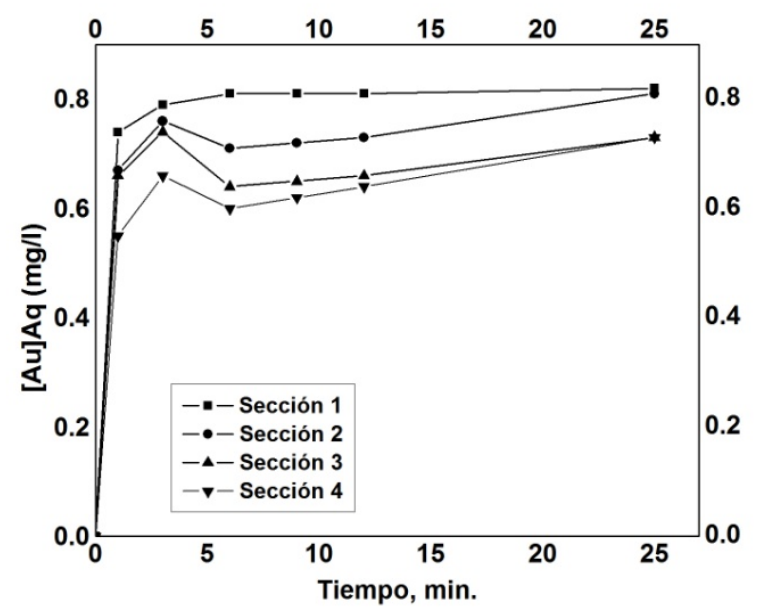

Fig. 9: Comportamiento en la extracción de oro al utilizar resina AuRIX ${ }^{\circledR} 100$ en las secciones de la columna a 0.70 , con flujo de $23 \mathrm{ml} / \mathrm{min}$, equivalente a $276 \mathrm{BVH}$

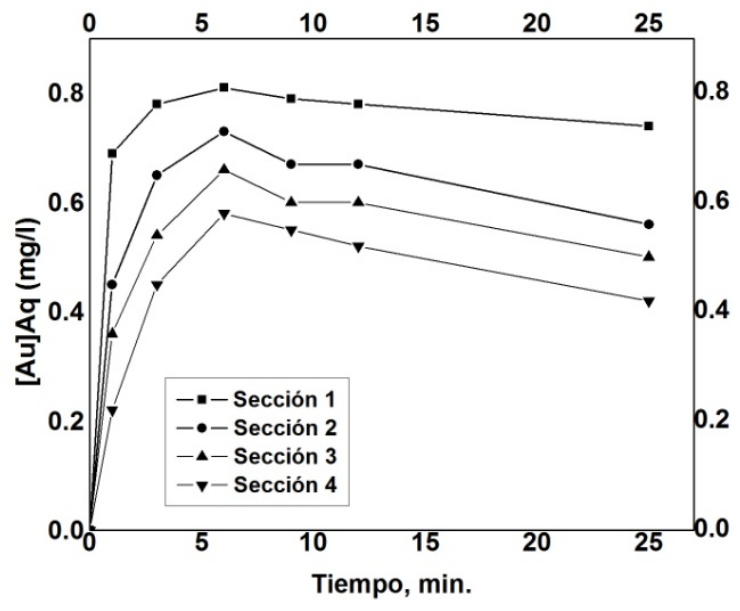

Fig. 8: Perfil de la concentración de oro, en las secciones de la columna con velocidad de 0.60 , con flujo de $15 \mathrm{ml} / \mathrm{min}$, equivalente a $180 \mathrm{BVH}$

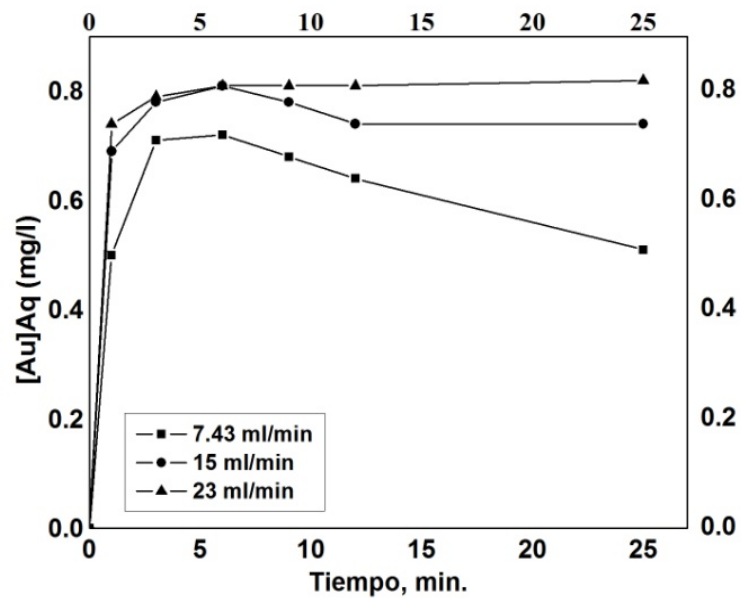

Fig. 10: Perfil de la concentración en la extracción de oro al utilizar resina AuRIX ${ }^{\circledR} 100$ de la primera sección de la columna manejando diferentes flujos

Para $180 \mathrm{BVH}$. se compararon las secciones se observó que se incrementa la velocidad de adsorción en cada sección con respecto a la velocidad mostrada en la Fig. 7 a 89 BVH. Se observó que la concentración de salida en cada sección se incrementa un poco con respecto a la de salida mostrada en la Fig. 7. Al aumentar el flujo aumenta la concentración de salida de la solución, esto indica que a mayor flujo el tiempo para alcanzar la concentración de saturación de la resina es menor y en caso de manejar flujos mayores sería necesario una quinta sección en la columna, para lograr la recuperación total del ión intercambiado.

\section{CONCLUSIONES}

En base a los resultados obtenidos del estudio de adsorción de oro, se puede concluir que: Al aumentar la concentración de tiosulfato de amonio de 0.00674, 0.03, 0.04 Molar se favoreció la extracción de oro, debido a una mayor presencia de tiosulfato libre. Cuando aumenta el valor de 
pH por encima de 11, los iones hidroxilo promueven la degradación de iones tiosulfato, causando la precipitación de oro.

Se encontró que la presencia de tiosulfato favorece la adsorción de oro. Con tiosulfato, se obtuvo en $0.04 \mathrm{M}$, se obtuvo un $99 \%$ de adsorción mientras que con $0.03 \mathrm{M}$ de tiosulfato, la adsorción disminuyó al $98 \%$, el proceso de adsorción fue más inestable y con $0.00674 \mathrm{M}$ la adsorción disminuyó al 98\%, pero las condiciones de adsorción fueron demasiado inestables.

La velocidad de agitación del sistema es una variable muy importante porque permite la suspensión de las partículas en movimiento y eso facilita la transferencia de masa. Las concentraciones de oro como afectan de 1, 2, y $8 \mathrm{mg} / \mathrm{l}$, los porcentajes de recuperación respectivamente fueron similares de 99, 93 y 95.75\% de recuperación de oro.

La resina AuRIX ${ }^{\circledR} 100$ tiene las ventajas de trabajar a pH altos típicos de las soluciones industriales (9-11.5), dando como resultado el $\mathrm{pH}$ mejor 10.5 y en $\mathrm{pH} 9$ disminuye la disolución de oro con tiosulfato de amonio, lo cual es atribuido a la inestabilidad en la disolución de oro.

\section{AGRADECIMIENTOS}

Los autores expresan sus agradecimientos a la Universidad de Sonora por brindar su apoyo durante la investigación, al Departamento de Ingeniería Química y Metalurgia, al Laboratorio de Servicios de Metalurgia, CONACyT, empresa COGNIS, a CESUES, a M.C. Juan Arévalo Amezcua, por su valiosa colaboración en la elaboración del proyecto.

\section{REFERENCIAS}

Aguayo, S., J.L. Valenzuela, J.R. Parga y M. Cruz, Continuous Laboratory Gold Solvent Extraction from Cyanide Solutions using LIX 79 Reagent, Chemical Engineering \& Technology 30(11), 14571599 (2007).

Aylmore, M.G. y D.M Muir, Thiosulfate leaching of gold-a review, Minerals Engineering 14, 135174 (2001).

Breuer, P.L. y M.I. Jeffrey, Electrochemical study of gold leaching in thiosulphate solutions containing ammonia and copper, Hydrometallurgy 65, 145-157 (2002).

Chaparro, M., "Extracción del complejo oro tiosulfato utilizando guanidina en una resina de intercambio aniónico", Tesis de Maestría, Dpto. de Ing. Química y Metalurgia, Univ. de Sonora, Sonora, México (2008).

Ficeriová, J., P. Baláž., E. Boldižarová y J. Stanislav, Thiosulfate leaching of gold from a mechanically activated CuPbZn concentrate, Hydrometallurgy 67(5), 37-43 (2002).

Jeffrey, M.I., D.M. Hewitt, X. Dai y S.D. Brunt, lon exchange adsorption and elution for recovering gold thiosulfate from leach solutions, Hydrometallurgy 100, 136-143 (2010).

Molleman, E. y D. Dreisinger, The treatment of copper-gold ores by ammonium thiosulfate leaching, Hydrometallurgy 66, 1-21 (2002).

Munive, T.G., M. Encinas, A. Valenzuela, Valenzuela J.L. y J.R. Parga, Estudio comparativo de la lixiviación de un mineral refractario con cianuro de sodio y tiosulfato de sodio para la recuperación de oro y plata, GEOMIMET 291, 12-24 (2011).

Navarro, P., C. Vargas, A. Villarroel y F.J. Alguacil, On the use of ammoniacal/ammonium thiosulphate for gold extraction from a concentrate, Hydrometallurgy 65, 37-42 (2002). 
Navarro, P., C. Vargas, V. Reveco y J. Orellana, Recuperación desde un medio amoniacotiosulfato con resina de intercambio iónico Amberlita IRA-410, Revista de Metalurgia 42(5), 354366 (2006).

Seob, I. y otros siete autores, Sequential process of sorption and incineration for recovery of gold from cyanide solutions: Comparison of ion exchange resin, activated carbon and biosorbent, Chemical Engineering Journal: 165, 440-446 (2010).

Valenzuela A., J.L. Valenzuela, S. Aguayo y J.R. Parga, Estudio de la resina AuRIX ${ }^{\circledR} 100$ de intercambio iónico para recuperar oro en las soluciones cianuradas, GEOMIMET (268), 26-38 (2006).

Vargas, C., P. Navarro, E. Araya, F. Pavez y F.J. Alguacil, Recuperación de oro a partir de disoluciones de amoniaco y tiosulfato utilizando carbón activado, Revista de Metalurgia 42 (3), 222-233 (2006).

Yen, W.T., M. Aghamirian, G. Deschenes y S. Theben, "Gold extraction from mild refractory ore using ammonium thiosulfate". Proceedings of the International Symposium on Gold Recovery, Montreal May 3-6 CIM (1998).

Zhang, H. y D.B. Dreisinger, The adsorption of gold and copper onto ion-exchange resins from ammoniacal thiosulfate solutions, Hydrometallurgy 66, 67-76 (2002). 